\title{
Fatigue life prediction of mechanical structures under stochastic loading
}

\author{
Bohuš Leitner ${ }^{1, *}$, Lucia Figuli ${ }^{1}$ \\ ${ }^{1}$ University of Žilina, Faculty of Security Engineering, Department of technical sciences \\ and informatics, 1. mája 32, Žilina, Slovakia
}

\begin{abstract}
Problems of fatigue life prediction of materials and structures are discussed in the paper. Service loading is assumed as a continuous loading process with possible discontinuous events, which are caused by various operating conditions. The damage in a material is due to a cumulative degradation process. The damaging process is then represented either by rain-flow matrices or by a fatigue damage function which is derived using some hypothesis of a fatigue failure criterion. Presented theoretical procedure enables a very effective estimation of a service life and/or reliable evaluation of residual life of any structures under various types of loading and environmental conditions. This approach creates a good basis for powerful expert systems in structural and mechanical engineering. The aim of the paper is to present briefly some results of analysis of load-bearing steel structure loads of special railway crane PKP 25/20i which was utilized in some specific ad relatively hard operating conditions. Virtual models of the structure were being used in an analysis of acting working dynamics loads influence to be able to forecast fatigue life of load-bearing of the crane jib.
\end{abstract}

Keywords: mechanical structures, stochastic loading, material degradation, fatigue damage, measurement of dynamics loads, fatigue life prediction, load-bearing structure

\section{Introduction}

Optimization of structure design is a decisive condition for effective production. It is exceptionally significant in mechanical and material engineering because either economy demands or waste effects could be considerable in this case. Progressive software (CAD and $\mathrm{CAE}$ ) techniques have an indisputable advantage compared to traditional empiricalexperimental methods because they are by far quicker and less expensive.

So that to accelerate calculation procedures, we must have a suitable databank of operating parameters and of their influence upon a service life. The parameters that affect a durability of structure parts are following: operating loading, structure characteristics, material properties and environmental conditions. Then we must use an appropriate method to estimate a service life and dependability for each combination of the parameters. Methods that enable these objectives are created by a combination of an empirical-

\footnotetext{
* Corresponding author: Bohus.Leitner@fbi.uniza.sk

Reviewers: Juraj Gerlici, Milan Vaško
} 
experimental, theoretic-analytical and computer-simulation approach. The fundamental problem is to respect a complete time loading history with possible non-homogeneous events.

Usually, the simulated loading is simultaneously transformed into closed hysteresis loops using a rain-flow method, and a damage accumulation is estimated according to failure hypotheses. In the case of composite materials, various material characteristics as well as material joints must be respected. That brings much more complications in comparison with procedures used for homogeneous materials.

\section{Theoretical background for operating conditions modelling}

The most serious problem in modelling of damage mechanics is to analyse and simulate operating conditions. The analysis and modelling of service conditions are the input operations in the simulation steps, according to Fig.1.

\begin{tabular}{|c|c|c|}
\hline \multicolumn{3}{|c|}{ MODELLING OF OPERATING CONDITIONS } \\
\hline V & & \\
\hline $\begin{array}{c}\text { Analysis of an } \\
\text { operating signal }\end{array}$ & $\begin{array}{c}\text { Transformation of the } \\
\text { process into a series of } \\
\text { closed cycles }\end{array}$ & $\begin{array}{c}\text { The model of a fatigue } \\
\text { damage mechanism }\end{array}$ \\
\hline $\begin{array}{l}\text { Measurement, identifica- } \\
\text { tion, evaluation, recon- } \\
\text { struction, modeling and } \\
\text { simulation of a loading } \\
\text { processes. }\end{array}$ & $\begin{array}{l}\text { A physical principle of } \\
\text { cycles closing (rain flow } \\
\text { counting, infor-mation of } \\
\text { a load history, successsi- } \\
\text { vity and time dimension }\end{array}$ & $\begin{array}{l}\text { Hypotheses of fatigue damage } \\
\text { cumulation, an influence of } \\
\text { loading, material and structure } \\
\text { parameters, operating conditions, } \\
\text { estimation of a fatigue damage } \\
\text { degradation. }\end{array}$ \\
\hline
\end{tabular}

Fig. 1. Diagram of modelling procedure

The correct simulation of service conditions is the necessary condition of a successful performance. Service conditions consist of following items [1]:

- operating loading (generally, random loading with discontinuous damaging events),

- material characteristics (endurance limit, ultimate strength, S-N curve, fatigue diagrams),

- structure parameters (notch stress concentrators, surface treatment, welds, ...),

- environmental properties (corrosion, friction, high/low temperatures, radiation, ...).

Operational loading. The analysis and simulation of the operating loading are the most important and complicated problems in the procedure. Methods how to perform the tasks are described in [2]. The methods summary is introduced in Fig. 2.

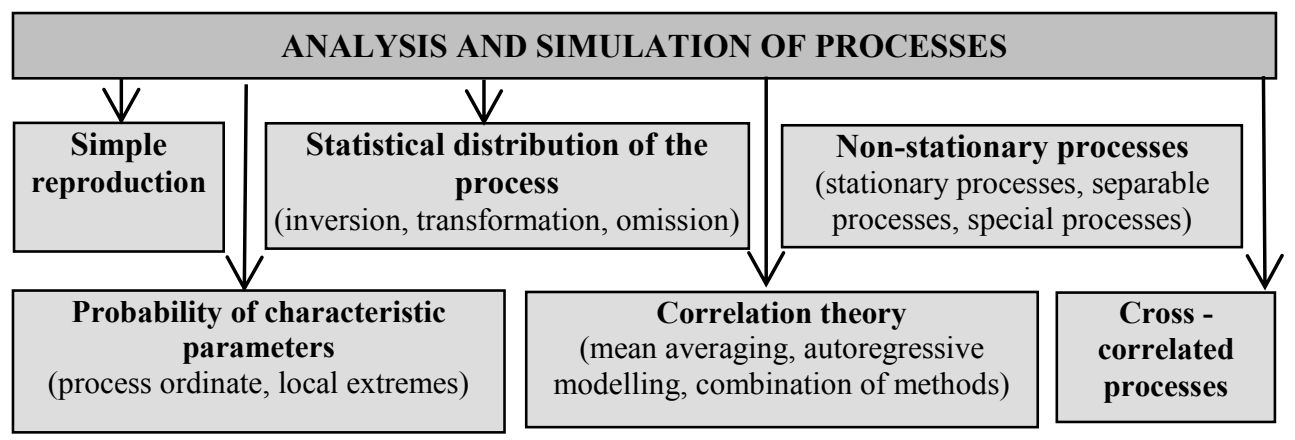

Fig. 2. Methods of a signal processing and generation 
An operating loading is mostly considered as a stationary or non-stationary stochastic process. Such a process is completely described by its both probability density function (including a mean value and variance) and power spectral density (auto-correlation function). However, various discontinuous events that are mostly caused by manoeuvres and special service conditions must be respected, too. This problem significantly complicates a simulation procedure, and new non-standard methods and algorithms must be applied [3]. It is also very important to have a reliable algorithm of counting of closed hysteresis loops. Rain-flow methods mostly render possible the procedure only after a definite block of closed loops and they suppose the knowledge of the entire time history before starting to count. In such a case, it is, however, possible neither continual monitoring of a fatigue damage after each cycle nor a running estimation of a residual life during an operation. The algorithm of a simultaneous signal generation and rain-flow counting was proposed in [1]. The method enables to respect a contribution of each closed hysteresis loop in the stress/strain domain continually, corresponding to the on-line generation of a loading process.

Material characteristics. Information of material characteristics of individual structure components is necessary for estimation of a fatigue damage. The most important characteristics are e.g. elastic modulus, shear modulus, strength limit, yield stress, fatigue limit, Woehler curve, cyclic deformation curve, etc. All the parameters must be identified before a simulating procedure, in order to be included into calculation algorithms. Characteristics for each structure component could be considered separately, what enables an investigation of composite structures. It is possible to suppose the time dependence of the characteristics, too. Therefore, we can also investigate composite materials with nonstationary properties.

Structure parameters. Simulated loading must be decomposed for the structure, according to the fatigue strength theory. In order to determine a real fatigue life curve, we must know structure characteristics, e.g. stress intensity factors, notch coefficients, surface sensitivities, size factors, welding parameters, etc. These parameters are then implicitly included in the actual endurance limit, and this way, into calculation relationship, too. In the case of multi-axial loading, some hypothesis of a stress recalculation must be applied. For example, the strain energy hypothesis for a combination of bending and torsion has form [1]

$$
\sigma_{e q}=\sqrt{\left(\sigma_{a}+\psi_{\sigma} \cdot \sigma_{m}\right)^{2}+3 \cdot\left(\tau_{a}+\psi_{\tau} \cdot \tau_{m}\right)^{2}}
$$

where $\sigma_{\mathrm{a}}$ is the amplitude of a normal stress, $\sigma_{\mathrm{m}}$ is the mean value of a normal stress, $\tau_{\mathrm{a}}$ is the amplitude of a shear stress, $\tau_{\mathrm{m}}$ is the mean value of a shear stress, and $\psi_{\sigma}, \psi_{\tau}$ are corresponding sensitivities to the asymmetry of a cycle.

Environmental properties. Parameters that correspond to environmental conditions have a serious influence upon a fatigue life, too. The factors are above all: corrosion, high or low temperatures, friction, radiation, etc. They could be also implicitly included into a calculation relationship. The advantage of this approach is again in the ability to consider possible non-stationary environmental characteristics [4]. That is very convenient for composite structures, too.

\section{Estimation of material degradation parameters}

Two basic methods for an estimation of a material degradation can be mostly used (Fig. 3). The first one is based on a rain-flow matrices creation, and according to the other method, we must specify a fatigue damage function. 
Rain-flow matrices of loading and damaging. The rain-flow matrix of loading (RML) is created by elements $l_{i, j}$ which represent the number of hysteresis loops that have been originated in the i-level and that have started their closing in the j-level [2].

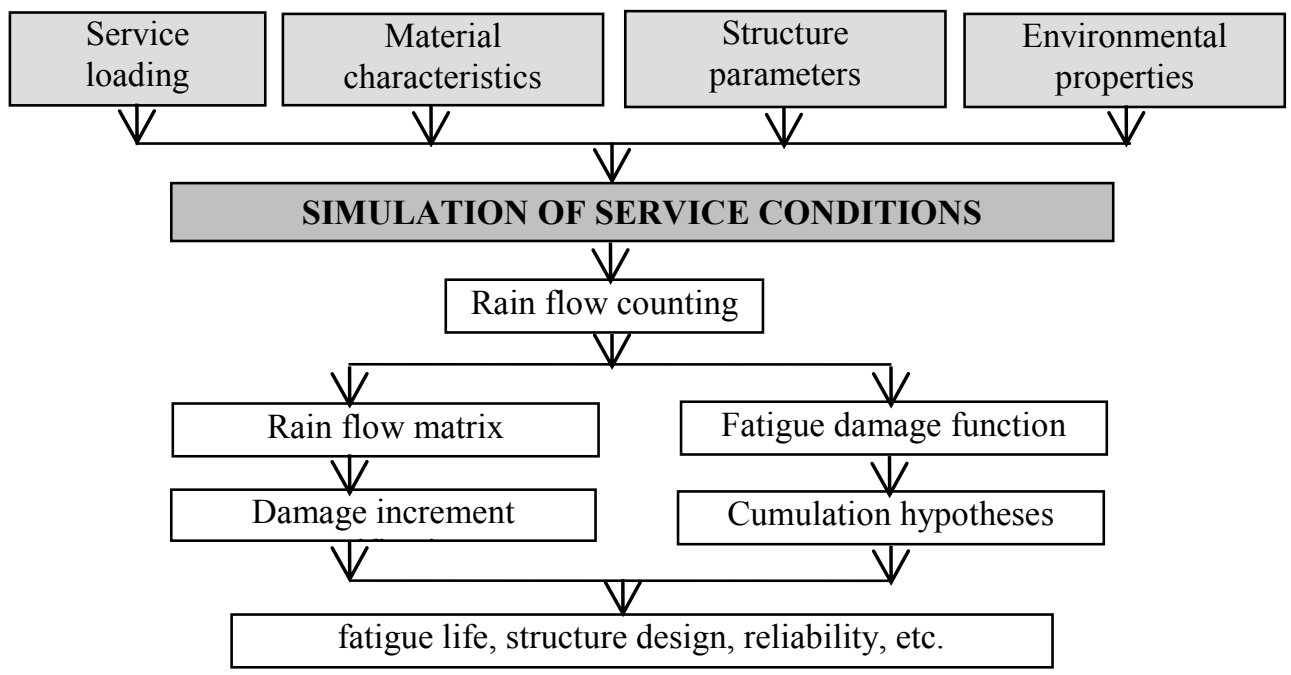

Fig. 3. The estimation procedure

In the case of non-stationary loading processes, and for non-linear hypotheses (or for cyclic unstable materials), the time moment of a given loop (or it's closing sequence) is also important, if need be an information about the foregoing hysteresis loop (or about more loops), too. Then, the rain-flow matrix must be extended by the next dimension, i.e. it must be a three-dimensional one. Each closed hysteresis loop produces some definite corresponding damaging influence. Indicating a damaging effect of the loop as $d_{i, j}$, we would obtain also a square rain-flow matrix of damaging (RMD) which is composed of the elements $d_{i, j}$. RMD could be three-dimensional, too. There is no problem, how to identify a damage quantity $d_{i, j}$, i.e. how to identify a damaging effect of a sole hysteresis loop. For this purpose, we must use some of hypotheses of a fatigue cumulative damage. In the case of a linear rule of fatigue damaging, we constitute two-dimensional RMD. Though, if we accept some non-linear hypothesis, the rain-flow matrix should be three-dimensional, too. Having both the RML and RMD, we can calculate the estimation of an accumulated fatigue damage $D$ as their scalar product [2], i.e.

$$
D=\sum_{i=1}^{n} \sum_{j=1}^{n} l_{i, j} \cdot d_{i, j} .
$$

Fatigue damage function. A creation of a fatigue damage function is the other possibility how to estimate degradation. Such a function is determined in the following form

$$
D_{n}\left(\sigma_{a}\right)=k\left(\sigma_{a}\right) \cdot n^{m\left(\sigma_{a}\right)} \quad ; \quad k\left(\sigma_{a}\right)>0 \quad ; \quad m\left(\sigma_{a}\right)>0
$$

where $k_{(\sigma a)}$ and $m_{(\sigma a)}$ are definite parameters which could be identified on the basis of fatigue damage criteria. The function depends both on the number of passed closed cycles and on the character of loading, i.e. on amplitudes and mean values of the cycles. Using this method, a continuous monitoring of a fatigue damage process is possible, and the time history is respected, too. The choice of the damage criterion is the problem in this case, because the well-known linear Miner's hypothesis is frequently not the optimal one, and other hypotheses must be adjusted to a special case, i.e. only the sole closed cycle is in each 
block of a macro-block, then. Frequently, the derivative function of a fatigue damage addition has the form

$$
d_{n}\left(\sigma_{a}\right)=k\left(\sigma_{a}\right) \cdot m\left(\sigma_{a}\right) \cdot n^{m\left(\sigma_{a}\right)-1},
$$

that corresponds to the above mentioned damaging effect of a definite closed loop.

Summing the function, the number of cycles till the critical damage accumulation $N_{k r}$ can be estimated according to the relationship [2]

$$
\sum_{n=1}^{N_{k r}} d_{n}\left(\sigma_{a}\right) \leq 1 \leq \sum_{n=1}^{N_{k r}+1} d_{n}\left(\sigma_{a}\right)
$$

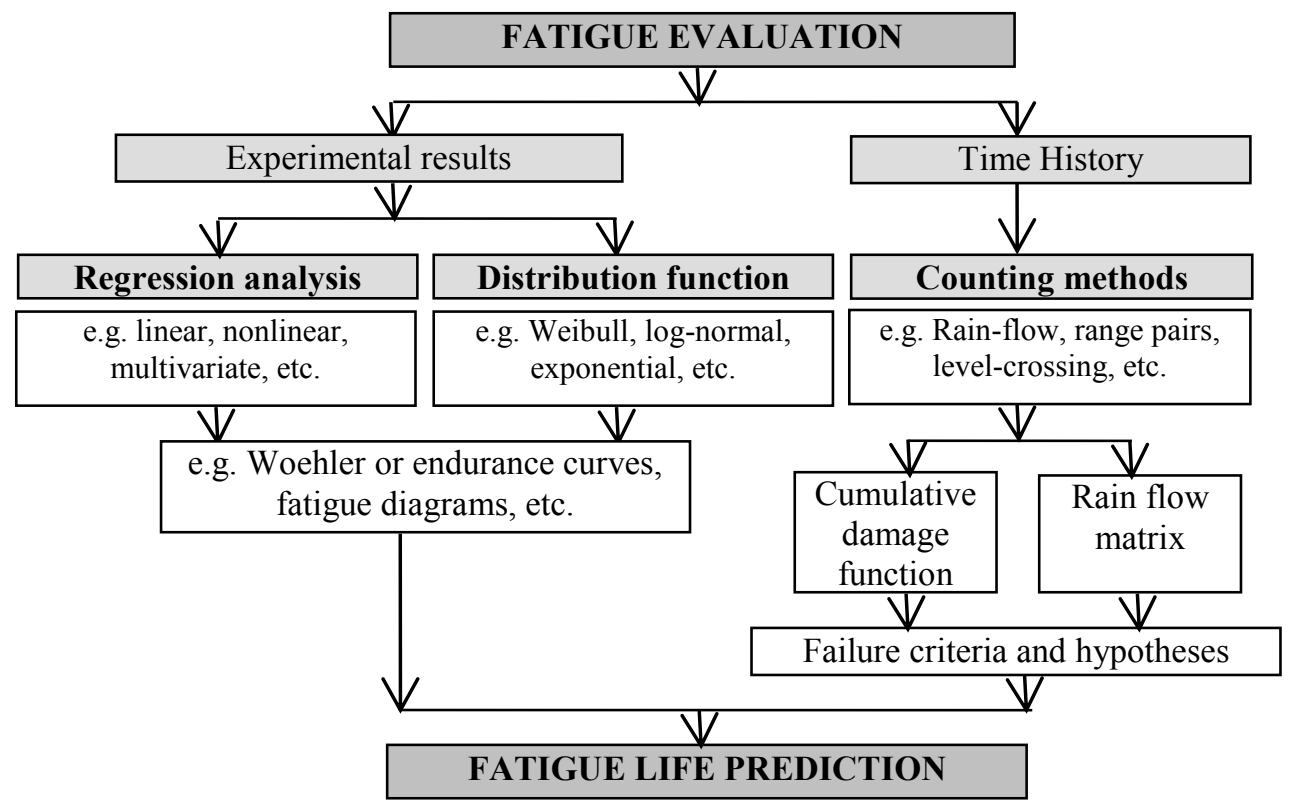

Fig. 4. The procedure of a fatigue life evaluation

\section{The computational scheme of a fatigue life prediction}

The procedure of a fatigue damage evaluation in order to predict a fatigue life is in Fig. 4. We can dispose either of experimental measured results or computer simulated data. Experimental data are processed using a regression analysis or a frequency distribution function is determined [5].

A selection of possible hypotheses is optional and it depends on a specific situation or on a philosophy of a fatigue damaging process. This way, a lot of tasks can be solved, e.g. an estimation of a fatigue life of a given structure under given loading, optimisation of a material choice, structure dimensioning, investigation of an environmental conditions influence, etc.

\section{Case study - prediction of fatigue life of load bearing structure}

A special railway crane PKP 25/20i is lifting equipment designed for exploitation in a limited space conditions which are met by the building of railway (Fig. 5). His main working purpose is to lay down or to tear off rail fields of maximal length $25 \mathrm{~m}$ and mass of 
20 tons by a complex reconstruction or by renovation of the railway. Determination of real working conditions with a choice of typical loads was important for calculation of fatigue life of a crane bridge as a carrying steel structure. There was possible to build a theoretical load spectrum of a crane load-bearing structure on that base.

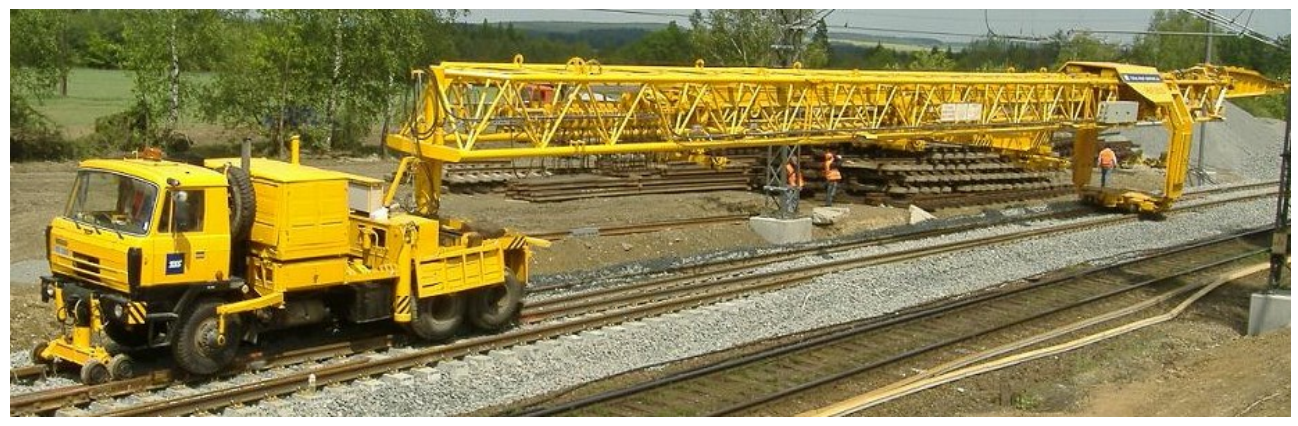

Fig. 5. A Special Railway Crane PKP 25/20i

It was necessary thoroughly analyse functions of the crane one by one and to determine how they influence the fatigue life by building of characteristic load spectrum of crane bridge. After a theoretical analysis of single working functions were following characteristic working functions of a crane:

- tearing off rail fields - the limiting function of a machine (high load of a crane bridge by high mass and dynamic effects by tearing off rail fields out of gravel bed),

- laying down rail fields - the limiting function of a crane,

- auxiliary working functions - manipulation of carriages, travel of a crab (type of complemented cycles, low influence on the total fatigue life of crane bridge),

- travel of a crane - function between basic technological workings (no important influence on fatigue damage of crane bridge),

It is obvious from the analysis of working conditions that the most exacting working conditions are the tearing off the old rail fields and that this function has the decisive influence on fatigue life from the point of view of number of cycles and loads.

\subsection{Selection of measuring conditions}

On the base of knowledge of fatigue life theory and analysis of working conditions was decided that an experimental measurement would be done in a real practice. The measurement was planned to realize by the renovation of a railway track. Before actual measurements there was necessary to determine critical locations of the crane bridge and to realize a theoretical analysis of working conditions of a crane. These were chosen on the base of static conditions calculations using model of FEM software package (Fig. 6).

The outputs of them were the locations for location of tensiometers on the crane bridge according to Fig.7. 




Analysis of measured data. By analysis of measured values of stresses was determined:

1. Stress by tearing off rail fields has a periodical character (Fig.8). The dynamic increase of stress was about 10-20\% of the maximal amplitude. The most expressive progress of stresses was determined during tearing off, start, stopping and lazing down rail fields. The measured value of stress amplitude was average $175 \mathrm{MPa}$.

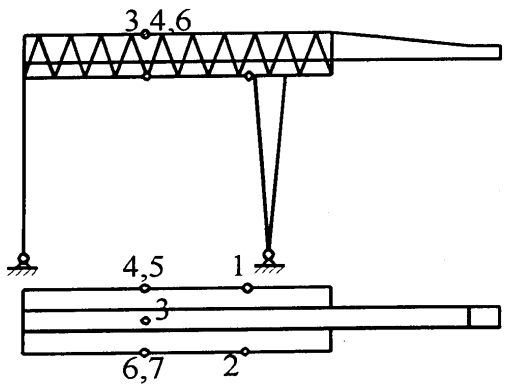

Fig. 7. Locations of tensiometers on a crane bridge



$-60$

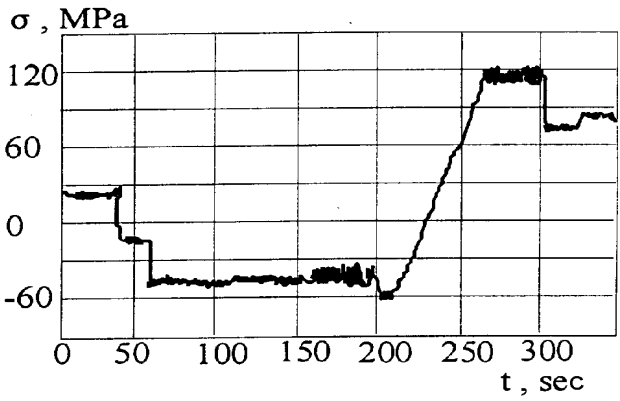

Fig. 8. Stress by tearing off, start, stopping and lazing down of rail fields

2. The manipulation with railway carriage (Fig.9) using turn able part of crane bridge was characterised by a non-stationary process by measured values of stress in average $20 \mathrm{MPa}$.

3. Travel of a crane to the next rail field was characterized by a non-stationary progress of stress increase, by which a maximal absolute value of stress increase was $34 \mathrm{MPa}$ (Fig 10).

For completeness in Tab.1 are the absolute values of the stresses in selected critical cross sections of the crane structure (obtained experimental measurements

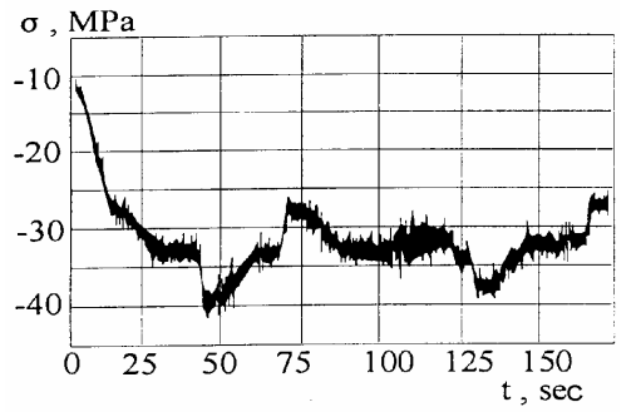

Fig. 9. Stress by manipulation using turntable part

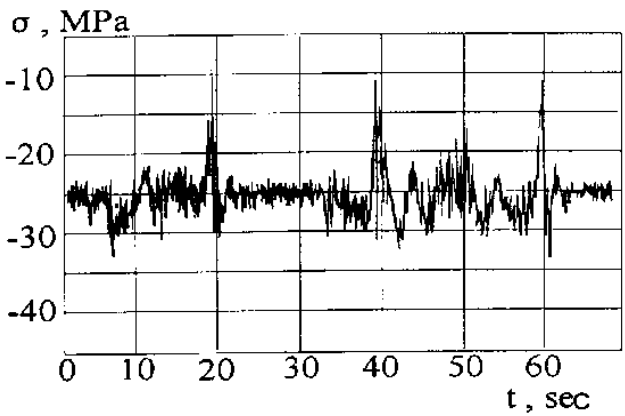

Fig. 10. Stress by travel to next rail field bridge 
Table 1. Tensions in the critical points of the structure

\begin{tabular}{|c|c|c|c|c|c|}
\hline \multirow{2}{*}{$\begin{array}{c}\text { Tensiometer } \\
\text { number }\end{array}$} & \multicolumn{5}{|c|}{ Measurement number [MPa] } \\
\cline { 2 - 6 } & $\mathbf{1}$ & $\mathbf{2}$ & $\mathbf{3}$ & $\mathbf{4}$ & $\mathbf{5}$ \\
\hline $\mathbf{1}$ & -85.22 & -93.33 & -87.27 & -104.91 & -95.62 \\
\hline $\mathbf{2}$ & -89.65 & -89.42 & -100.10 & -94.76 & -87.71 \\
\hline $\mathbf{3}$ & 139.12 & 165.37 & 151.09 & 153.68 & 154.32 \\
\hline $\mathbf{4}$ & 176.29 & 165.27 & 170.62 & 176.46 & 175.83 \\
\hline $\mathbf{5}$ & 135.77 & 150.54 & 140.65 & 147.62 & 138.96 \\
\hline $\mathbf{6}$ & 170.51 & 150.99 & 165.96 & 172.43 & 170.83 \\
\hline $\mathbf{7}$ & 139.15 & 152.33 & 137.92 & 147.49 & 144.89 \\
\hline
\end{tabular}

Spectrum of loads. On the base of an analysis of working conditions and results of experimentally measured increase of stresses during working of a crane was possible to construct a real spectrum of loads (Fig.7). Single working cycles introduce characteristically working conditions of a railway crane, which consist of following working cycles:

- $A$ - tearing off old rail fields $(\sigma=175 \mathrm{MPa}, n=1250)$

- $C$ - auxiliary working cycles

$C 1$ - free travel of crabs ( $\sigma=40 \mathrm{MPa}, n=3000$ cycles $)$,

$C 2$ - travel to the next rail field ( $\sigma=34 \mathrm{MPa}, n=i$ cycles),

$C 3$ - manipulation with of railway carriage $(\sigma=20 \mathrm{MPa}, n=650$ cycles $)$.

\subsection{Fatigue Life Prediction of Crane Load-Bearing Structure}

The general procedure of life prediction from point of view of fatigue damage was based:

- determination of the most significant working regimes and factors of typical working conditions from point of view of structure loads and their following activities [5-7],

- identification of carrying structure critical parts and determination of their working loads,

- analysis of choice material properties and estimate of effects of different types of notches, connections and nonlinearities in examined points of structure $[8,9]$ and

- application of suitable hypothesis of fatigue damage cumulation and quantification of predicted fatigue life of selected parts of carrying structure [10,11].

The most significant working regimes. The activities connected with technology of tearing off the old rail fields and laying down the new ones were identified as the most significant working regimes of mentioned crane and some of accessory processes as noload run of a crab, handling of railway bogies and travelling to the following rail field too. The effect of surroundings low temperatures was chosen from surrounding conditions because they cause freezing of the rails bed and following increase of the pulling force by tearing off rail fields. The effects of surroundings conditions connected with unevenness (longitudinal or transverse) of newly laid down rail bands were not taken into account because it has not an expressive effect on crane working.

The identification of critical parts of structure. The virtual FEM model was elaborated and verified to use in the procedure of identification of supporting structure critical parts. The stresses experimentally obtained were utilized during the actualization of the FEM model in order to obtain the maximum adequacy of the model comparing to the real structure. It was determined from static and dynamic analyses realized. The critical parts 
are namely upper and lower beams of supporting structure (EL232, EL237, EL89 and EL93) and places near of locking connection of C and D parts (EL335) on (Fig.11).

Material properties of structure details. Supporting structure of the crane bridge is made from the low carbon steel 11 523. The Woehler curve of the used material was determined after the STN standards. Obtained Woehler curve of the load/bearing structure material and its parameters were utilized as an input into the suitable hypothesis of fatigue damage cumulation $\left(w=5,8, N_{C}=2,5.106, \sigma_{C}=190 \mathrm{MPa}\right.$ and $\left.R_{e}=355 \mathrm{MPa}\right)$.

Prediction of working fatigue life of a load-bearing structure. The principal hypotheses of fatigue damage cumulation were applied during prediction of working fatigue life of a crane supporting structure. It was made by means of quantification of cumulation fatigue damage process too. Three principal hypotheses of fatigue damage cumulation were applied. There were used the Palmgren-Miner (P-M) and the Corten-Dolan (C-D) hypothesis which are based on utilization of corresponding material curve and actual loading spectrum.



Fig. 11. Detail of $C$ and $D$ parts in locking connection

Rajcher's theorem defines the fatigue damage in the critical location of the structure part induced per one second and is expressed by the following equation in form [1]

$$
D_{s}=\frac{\Gamma \cdot\left(\frac{w}{2}+1\right) \cdot\left[2 \cdot \int_{0}^{\infty} f^{\frac{2}{w}} \cdot S(f) d f\right]^{\frac{w}{2}}}{N_{C} \cdot \sigma_{C}^{w}}
$$

where $w$ is exponent of $\mathrm{S} / \mathrm{N}$ curve, $\sigma_{c}$ is fatigue limit, $N_{c}$ is limit number of cycles to failure, $f$ is frequency, $S(f)$ is spectral power density of the stress loading process, $\Gamma$ is gamma function value. Time until the next failure (in hours) can be expressed as follows

$$
T=\frac{1}{3600 \cdot D_{s}}=\frac{N_{C} \cdot \sigma_{C}^{w}}{3600 \cdot f_{e} \cdot\left(2 \cdot s_{\sigma}^{2}\right)^{\frac{w}{2}}} \cdot \Gamma \cdot\left(\frac{w}{2}+1\right) .
$$

It is obvious that all the process can be realized only by means of the computer technique efficient enough. The approach in practice is that after import or calculation of the process spectral power density values $S(f)$ the process standard deviation $S_{\sigma}$ and process effective frequency $f_{e}$ of the probability density will be determined. The practical 
application of the presented process was realized by the program created in the MATLAB ${ }^{\odot}$ environment. The worked computational program named FATIGUE.M was used at the fatigue life computational estimation in the selected critical points under the chosen characteristics of the crane operating conditions [6]. The Rajcher's hypothesis of fatigue damage cumulation uses Woehler curve parameters, Gamma function and power spectral density (PSD) of a structure response an acting working loads [11]. Obtained results of estimated measure of load-bearing structure fatigue life are in Tab.2.

Table 2. The estimate of fatigue life of critical elements of crane carrying structure

\begin{tabular}{|l|c|c|c|}
\hline $\begin{array}{l}\text { Fatigue life prediction based } \\
\text { on hypothesis' [in years] }\end{array}$ & $\begin{array}{c}\text { Element } \\
\mathbf{2 3 2}\end{array}$ & $\begin{array}{c}\text { Element } \\
\mathbf{8 9}\end{array}$ & $\begin{array}{c}\text { Element } \\
\mathbf{3 3 5}\end{array}$ \\
\hline P-M (Palmgren-Miner) & 24.2 & 23.1 & 25.0 \\
\hline C-D (Corten - Dolan) & 19.6 & 19.2 & 21.2 \\
\hline R (Rajcher) & 20.2 & 19.9 & 20.4 \\
\hline
\end{tabular}

Demand for this procedure was made by its user. The term of life recommended by producer was 15 years by acceptation of determined technical conditions and recommendations. It was possible to declare basing on results obtained that it is possible to utilize the crane after proposed term of technical life from the point of view of fatigue life. However, the preventive checks of critical points were recommended in each 2-yers interval.

\section{Conclusions}

It follows from obtained results from point of view of safety and reliability of the crane PKP 25/20i working that its supporting structure - bridge of a crane is designed sufficiently. But it is necessary to respect proposed technology of its utilization and maximum value of loads. However, the non-reversible deformation and structure damage can occur during certain working conditions (for example tearing off rail field from frozen bed, strong wind impact or non-qualified manipulation and control resulting in the loss of crane stability). It is necessary to state finally that the fatigue damage cumulation process and fatigue life estimation can be made with certain probability only using certain assumptions and simplification despite of having available lot of data from the areas of fatigue strength.

This work has been supported by project VEGA No. 1/0240/15.

\section{References}

1. M. Růžička, M., Hanke, M., Rost, Dynamic Strength and Lifetime [in Czech]. (CVUT, Prague, 1989)

2. J. Čačko, Modelling of a Fatigue Cumulative Damage under Random Loading Conditions. Fracture Mechanics: Applications and Challenges, Elsevier, Amsterdam 2000, 113-120 (2000)

3. A. Sorg, J. Utzinger, B. Seufert, M. Oechsner, Fatigue life estimation of screws under multiaxial loading using a local approach. International Journal of Fatigue 104, 43-51

4. A. Chovanec, Analysing and modelling off-road vehicle availability. Transport Means: proceedings of the 16th International conference, October 25-26, 2012, Kaunas University of Technology, Lithuania, 54-57 (2012)

5. P. Kopas, L. Jakubovičová, M. Vaško, M. Handrik, Fatigue resistance of reinforcing steel bars. Proceedia Engineering 136, 193-197 (2016) 
6. M. Vaško, B. Leitner, M. Sága, Computational fatigue damage prediction of the lorry frames under random excitation. Communications 12 (4), 62-67 (2010)

7. J. Gerlici, T. Lack, Railway wheel and rail head profiles development based on the geometric characteristics shapes. Wear 271 (1-2), 246-258

8. J. Dižo, M. Blatnický, Use of multibody system dynamics as a tool for rail vehicle behaviour diagnostics. Diagnostyka 17 (2), 9-16 (2016)

9. Q. Dong, G. N. Xu, H. L. Ren, A. H. Wang, Fatigue remaining life estimation for remanufacturing truck crane Jib structure based on random load spectrum. Fatigue and Fracture of Engineering Materials and Structures 40 (5), 706-731

10. M. Vaško, M. Blatnický, P. Kopas, M. Sága, Research of weld joint fatigue life of the AlMgSi07.F25 aluminium alloy under bending-torsion cyclic loading. Metalurgija 56 (1-2), 94-98 (2017)

11. M. Sága, M. Vaško, P. Pecháč, Chosen numerical algorithms for interval finite element analysis. Procedia Engineering 96, 400-409 (2014) 